\title{
Video Article \\ Artificial Antigen Presenting Cell (aAPC) Mediated Activation and Expansion of Natural Killer T Cells
}

\author{
James E. East ${ }^{* 1}$, Wenji Sun* ${ }^{* 1}$, Tonya J. Webb ${ }^{1}$ \\ ${ }^{1}$ Department of Microbiology and Immunology, University of Maryland \\ * These authors contributed equally
}

Correspondence to: Tonya J. Webb at twebb@som.umaryland.edu

URL: https://www.jove.com/video/4333

DOI: doi:10.3791/4333

Keywords: Immunology, Issue 70, Medicine, Molecular Biology, Cellular Biology, Microbiology, Cancer Biology, Natural killer T cells, in vitro expansion, cancer immunology, artificial antigen presenting cells, adoptive transfer

Date Published: $12 / 29 / 2012$

Citation: East, J.E., Sun, W., Webb, T.J. Artificial Antigen Presenting Cell (aAPC) Mediated Activation and Expansion of Natural Killer T Cells. J. Vis. Exp. (70), e4333, doi:10.3791/4333 (2012).

\section{Abstract}

Natural killer T (NKT) cells are a unique subset of T cells that display markers characteristic of both natural killer (NK) cells and T cells ${ }^{1}$. Unlike classical T cells, NKT cells recognize lipid antigen in the context of CD1 molecules ${ }^{2}$. NKT cells express an invariant TCRa chain rearrangement: Va14Ja18 in mice and Va24Ja18 in humans, which is associated with V $\beta$ chains of limited diversity ${ }^{36}$, and are referred to as canonical or invariant NKT (iNKT) cells. Similar to conventional T cells, NKT cells develop from CD4-CD8- thymic precursor T cells following the appropriate signaling by CD1d ${ }^{7}$. The potential to utilize NKT cells for therapeutic purposes has significantly increased with the ability to stimulate and expand human NKT cells with $\alpha-$-Galactosylceramide ( $\alpha-G a l C e r)$ and a variety of cytokines ${ }^{8}$. Importantly, these cells retained their original phenotype, secreted cytokines, and displayed cytotoxic function against tumor cell lines. Thus, ex vivo expanded NKT cells remain functional and can be used for adoptive immunotherapy. However, NKT cell based-immunotherapy has been limited by the use of autologous antigen presenting cells and the quantity and quality of these stimulator cells can vary substantially. Monocyte-derived DC from cancer patients have been reported to express reduced levels of costimulatory molecules and produce less inflammatory cytokines ${ }^{9,10}$. In fact, murine DC rather than autologous APC have been used to test the function of NKT cells from CML patients ${ }^{11}$. However, this system can only be used for in vitro testing since NKT cells cannot be expanded by murine DC and then used for adoptive immunotherapy. Thus, a standardized system that relies on artificial Antigen Presenting Cells (aAPC) could produce the stimulating effects of DC without the pitfalls of allo- or xenogeneic cells ${ }^{12,13}$. Herein, we describe a method for generating CD1d-based aAPC. Since the engagement of the T cell receptor (TCR) by CD1d-antigen complexes is a fundamental requirement of NKT cell activation, antigen: CD1d-lg complexes provide a reliable method to isolate, activate, and expand effector NKT cell populations.

\section{Video Link}

The video component of this article can be found at https://www.jove.com/video/4333/

\section{Protocol}

\section{Generation of aAPC}

1. Before adding proteins to beads, prepare all reagents and buffers: $0.1 \mathrm{M}$ Borate buffer; $1 \mathrm{X}$ D-PBS (no $\mathrm{Ca}^{2+}$ and $\mathrm{Mg}^{2+}$ ); $\mathbf{B e a d}_{\text {Wash } \mathrm{Buffer}}$ ( 1 X PBS $+5 \%$ Human $A B$ serum $+0.02 \%$ sodium azide); Complete medium (RPMI medium $+100 \mathrm{mM}$ sodium pyruvate, $10 \mathrm{mM}$ non-essential vitamin solution, $100 \mathrm{mM}$ MEM Vitamin solution, 1\% 2-mercaptoethanol, $10 \mu \mathrm{M}$ ciprofloxacin, $5 \%$ Human AB serum); MACS buffer (1 L PBS free of $\mathrm{Ca}^{2+}$ and $\mathrm{Mg}^{2+}, 5 \mathrm{~g} \mathrm{BSA}$, and $2 \mathrm{mmol}$ EDTA).

2. Rinse $1 \mathrm{ml}$ Dynabeads M-450 Epoxy beads with $3 \mathrm{ml}$ sterile $0.1 \mathrm{M}$ Borate buffer (boric acid and water, $\mathrm{pH} 7.0-7.4$ ) in a $5 \mathrm{ml}$ clear borosilicate glass threaded vial.

3. In a separate $1.5 \mathrm{ml}$ microcentrifuge tube, add $100 \mu \mathrm{g}$ hCD1d-Ig dimer and $20 \mu \mathrm{g}$ costimulatory molecules (example: anti-CD28mAb) to $1 \mathrm{ml}$ PBS w/o $\mathrm{Ca}^{2+}$ or $\mathrm{Mg}^{2+}$

4. Place bead containing glass vial on magnet and aspirate borate buffer from beads. Add protein mixture from step 1.2 to glass vial and replace cap. Mix immediately by inverting the vial, cover the cap with parafilm, and place on a rotator and incubate overnight at $4{ }^{\circ} \mathrm{C}$.

5. The next day, place glass vial on magnet and remove protein mixture, while carefully avoiding beads. Wash the beads by adding $3 \mathrm{ml}$ bead wash buffer (PBS with $5 \% \mathrm{AB}$ serum $+0.02 \%$ sodium azide), and incubating at $4{ }^{\circ} \mathrm{C}$ on a rotator for 5 min. Repeat twice.

6. Beads can be stored in this bead wash mixture. To make functional aAPC, remove a small aliquot and count the beads using a hemacytometer. Check that the proteins are stably loaded onto the beads by staining with antibodies (ex. PE- conjugated anti-mouse lgG1) and performing flow cytometric analyses.

7. To load beads with antigen, remove $5 \times 10^{7}$ beads, and add to a small $1.5 \mathrm{ml}$ glass vial, rinse beads with $1 \mathrm{ml}$ sterile PBS. Resuspend washed beads with $1 \mathrm{ml}$ sterile PBS and add antigen; example: Load with $\alpha$-GalCer/KRN7000 (5mg/ml). * Note: While, we have not detected any issues with lipid solubility or micelle formation, lipids should be handled according to manufacturer's recommendations. Specifically, 
KRN7000 was reconstituted in DMSO $(1 \mathrm{mg} / \mathrm{ml})$ for these studies. KRN7000 and other glycolipid antigens can also be dissolved in $0.2 \mathrm{mg} / \mathrm{ml}$ PBS containing $0.5 \%$ Tween-20 (sonicate $2 \mathrm{hr}$. at $37^{\circ} \mathrm{C}$ ). IMPORTANT- The aAPC need to be loaded for at least $48-72 \mathrm{hr}$ prior to use.

\section{Isolation of $\mathrm{CD} 161^{+} \mathrm{CD}^{+}$Cells}

1. Collect peripheral blood mononuclear cells (PBMC). For Ficoll density gradient centrifugation separation of lymphocytes from a buffy coat or leukopheresis pack, first dilute heparinized blood with an equal volume of $1 \mathrm{X}$ PBS at room temperature.

2. Add $15 \mathrm{ml}$ of Ficoll (warmed to room temperature) to $50 \mathrm{ml}$ conical tubes. Slowly overlay $25 \mathrm{ml}$ of the diluted blood mixture on top of the Ficoll. Centrifuge at 2,000 rpm for 30 min at room temperature with the brake off.

3. Carefully remove the lymphocyte interface (white ring between the media and Ficoll) with a Pasteur pipette and transfer to a new $50 \mathrm{ml}$ conical tube.

4. Wash the cells by filling up the tube to $50 \mathrm{ml}$ with PBS and centrifuging at $1,500 \mathrm{rpm}$ for $5 \mathrm{~min}$. Discard the supernatant and combine the tubes from a single individual to a single tube and wash the peripheral blood mononuclear cells (PBMC) again with $20 \mathrm{ml}$ PBS. Then count the PBMC and resuspend at a concentration of $5 \times 10^{7}$ cells $/ \mathrm{ml}$ in MACS buffer ( $1 \mathrm{~L} \mathrm{PBS}$ free of $\mathrm{Ca}^{2+}$ and Mg ${ }^{2+}, 5 \mathrm{~g} \mathrm{BSA}$, and $2 \mathrm{mmol}$ EDTA).

5. In order to isolate the T cell fraction, start with $2 \mathrm{ml}$ of PBMC ( $10^{8}$ cells) and add $100 \mu \mathrm{l}$ of Pan T cell enrichment solution from the EasySep Human T Cell Enrichment Kit. Incubate at room temperature for 10 min.

6. Add $100 \mu \mathrm{l}$ of magnetic particles to the solution and incubate at room temperature for another $10 \mathrm{~min}$. Bring the final volume of solvent to 2.5 $\mathrm{ml}$ and place the tube in the purple magnet for $5 \mathrm{~min}$. Quickly pour off the $\mathrm{CD}^{+}$fraction into a $15 \mathrm{ml}$ conical tube.

7. Wash the cells by adding $5 \mathrm{ml}$ cold MACS buffer, count the number of viable cells, and remove an aliquot for FACS staining.

8. To select the CD $161^{+}$cells, first resuspend enriched T cells in $980 \mu \mathrm{l}$ ice cold MACS buffer, add $10 \mu \mathrm{g}$ anti CD161 mAb, and incubate in refrigerator for $10 \mathrm{~min}$.

9. Centrifuge the cells at $1,500 \mathrm{rpm}$ at $4{ }^{\circ} \mathrm{C}$ for $5 \mathrm{~min}$. Then reconstitute the cell pellet in $800 \mu \mathrm{l}$ of MACS buffer. Add $200 \mu \mathrm{l}$ of anti-mouse lgG1 microbeads and incubate the solution for $10 \mathrm{~min}$ at $4{ }^{\circ} \mathrm{C}$.

10. During this incubation step, equilibrate a LS column by adding $3 \mathrm{ml}$ MACS buffer.

11. Next, wash the cells by centrifuging $1,500 \mathrm{rpm}$ at $4{ }^{\circ} \mathrm{C}$ for $5 \mathrm{~min}$. Resuspend the cells in $3 \mathrm{ml}$ MACS buffer. Then pipette the cells into the LS MACS separating column. Make sure to avoid generating bubbles by pipetting slowly. Rinse the column by adding $3 \mathrm{ml}$ of MACS buffer. Repeat twice.

12. Add $3 \mathrm{ml}$ fresh MACS buffer and remove column from magnet. Place column into a $15 \mathrm{ml}$ conical tube. Insert plunger and push out contents to obtain purified $\mathrm{CD} 161^{+} \mathrm{CD}^{+}$cells. Count NKT cell enriched fraction. You should have 2-4 million cells.

\section{3. aAPC-mediated NKT Cell Expansion}

1. Set up co-culture by adding $10^{6}$ enriched $\mathrm{CD} 161^{+} \mathrm{CD} 3^{+} \mathrm{T}$ cells and $10^{6} \mathrm{aAPC}$ in $16 \mathrm{ml}$ complete medium (complete medium $+\mathrm{IL}-2,100 \mathrm{U} / \mathrm{ml}$ ). Plate this mixture by adding $160 \mu \mathrm{l} /$ well final volume to a 96 well tissue-culture treated polystyrene, U-bottom plate with low-evaporation lid. Perform medium exchange every $7^{\text {th }}$ day by adding $80 \mu$ l of fresh medium.

2. Harvest cells, count, and perform FACS staining on day 12-14.

3. The expanded NKT cells can be replated as described above in step 3.1, specifically resuspend $10^{6}$ expanded T cells and $10^{6}$ aAPC in 16 ml complete medium. Plate this mixture by adding $160 \mu \mathrm{l} /$ well to a 96 well U-bottom plate. Continue to refresh coculture medium every $7^{\text {th }}$ day by adding $80 \mu \mathrm{l}$ of fresh medium.

4. It is best to freeze extra NKT cells following the second round of expansion ( $1 \times 10^{6} /$ cryovial in $1 \mathrm{ml}-5 \% \mathrm{DMSO} / 95 \% \mathrm{FBS}$.)

\section{Functional Test: aAPC-mediated Stimulation of NKT Cells}

1. Set up $5 \times 10^{4} \mathrm{NKT}$ cells/ well with $5 \times 10^{5}$ aAPC in $200 \mu \mathrm{l}$ final volume (complete medium) in 96 well U-bottom plate.

2. Harvest cell culture supernatant for ELISA after $24-48 \mathrm{hr}$.

\section{Representative Results}

Herein we describe a method for generating CD1d-lg based aAPC, made by covalent coupling of CD1d-lg and anti-CD28 mAb to magnetic beads to stimulate NKT cells as a standardized method for the propagation of NKT cells (Figure 1).First, one must demonstrate that the CD1d-Ig fusion proteins are stably immobilized onto the surface of the magnetic beads. As shown in Figure 2A, CD1d-Ig and anti-CD28 antibodies were both expressed on the surface of the magnetic beads. To examine the stimulatory capacity of the aAPC, we co-cultured NKT cell hybridomas with aAPC overnight, harvested the culture supernatants and measured IL-2 production by ELISA. We found that CD1d-Ig based aAPC were able to stimulate the NKT cell hybridomas at levels equal to or higher than their cellular counterparts (Figure 3, data not shown). Interestingly, we found that our mouse NKT cell hybridomas are stimulated by human CD1d-based aAPC (Figure 2B), which provides simple method for testing each batch of aAPC.

Next, we sought to demonstrate the propagation potential of aAPC, thus human T cells were isolated from the peripheral blood. First the $\mathrm{CD} 161^{+} \mathrm{CD}^{+} \mathrm{T}$ cell fraction was enriched by magnetic bead separation. Then, the T cells were stimulated biweekly with $\alpha-G a l C e r-l o a d e d ~ a A P C$. Importantly, we found that even with a relatively low initial NKT cell population $(0.03 \%)$, we were able to expand the cells to $\sim 67 \%$ Va24 ${ }^{+} V \beta 11^{+}$ (Figure 3). We have expanded NKT cells from the PBMC of many healthy volunteers and cancer patients and have found that $\alpha-G a l C e r$ loaded aAPC were able to expand the NKT cells population in both groups. Notably, the expansion rate was highly donor dependent. As expected the higher the initial population of $\mathrm{Va} 24^{+}$cells, the greater the percentage of expansion. In addition, when using a starting population of 2 million cells $\mathrm{CD} 161^{+} \mathrm{CD} 3^{+} \mathrm{T}$ cells, one can obtain $>10^{7}$ cells after two rounds of expansion (Table 1). Approximately $80-90 \%$ of the expanded NKT cells are $\mathrm{CD} 4^{+}, \sim 5 \% \mathrm{CD}^{+}$, and the remaining are presumably CD4-CD8- double negative NKT cells. These expanded NKT cells can be used for functional studies as shown in Figure 4A-C. We have found that our ex vivo expanded NKT cells remain responsive to a-GalCer stimulation and are potent producers of IL-17A, TNF- $\alpha$, and IFN-y. It should be noted that if the initial T cell enrichment population is low and one is unable 
to perform the second CD161 enrichment step, the aAPC-mediated expansion may not yield the expected results (see Figure 4D, Donor 1). However, if the percentage of circulating NKT cells is higher than $0.1 \%$, one should still be able to obtain a significant expansion of $i$ NKT cells. Collectively, these data demonstrate that CD1d based-aAPC can be used to effectively expand and stimulate primary human NKT cells.

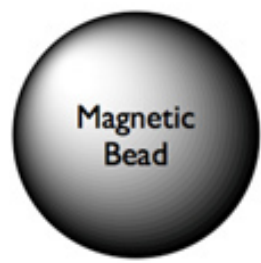

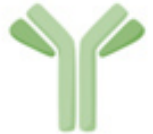

anti-CD28 antibody

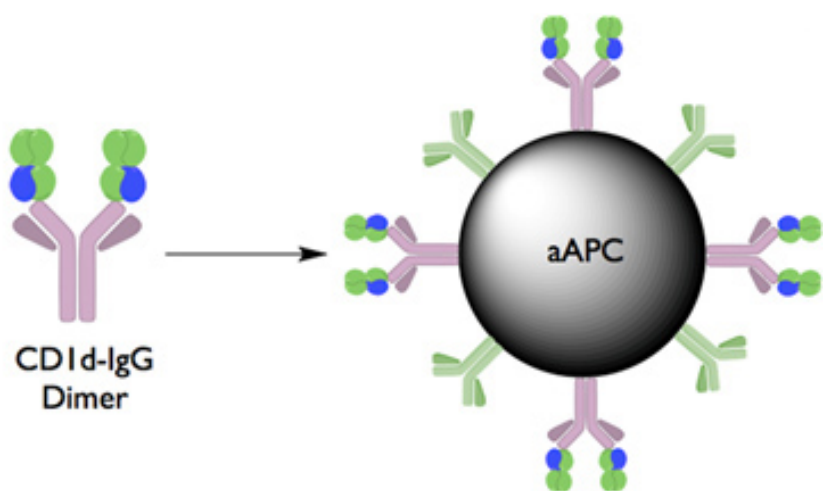

Figure 1. Schematic diagram of CD1d:Ig-based aAPCs Extracellular portions of the CD1d molecule are fused to the constant region of an immunoglobulin heavy chain protein separated by a short amino acid linker. These molecules can be easily loaded with lipid antigens, such as $\alpha-G a l C e r$, simply by incubating them with an excess of the lipid of interest. aAPC were made by coupling CD1d-lg and anti-CD Abs to magnetic beads. In this system, CD1d-Ig is used to provide the cognate antigen-specific signal through the TCR and anti-CD28 mAb provides the costimulatory signal.

A.
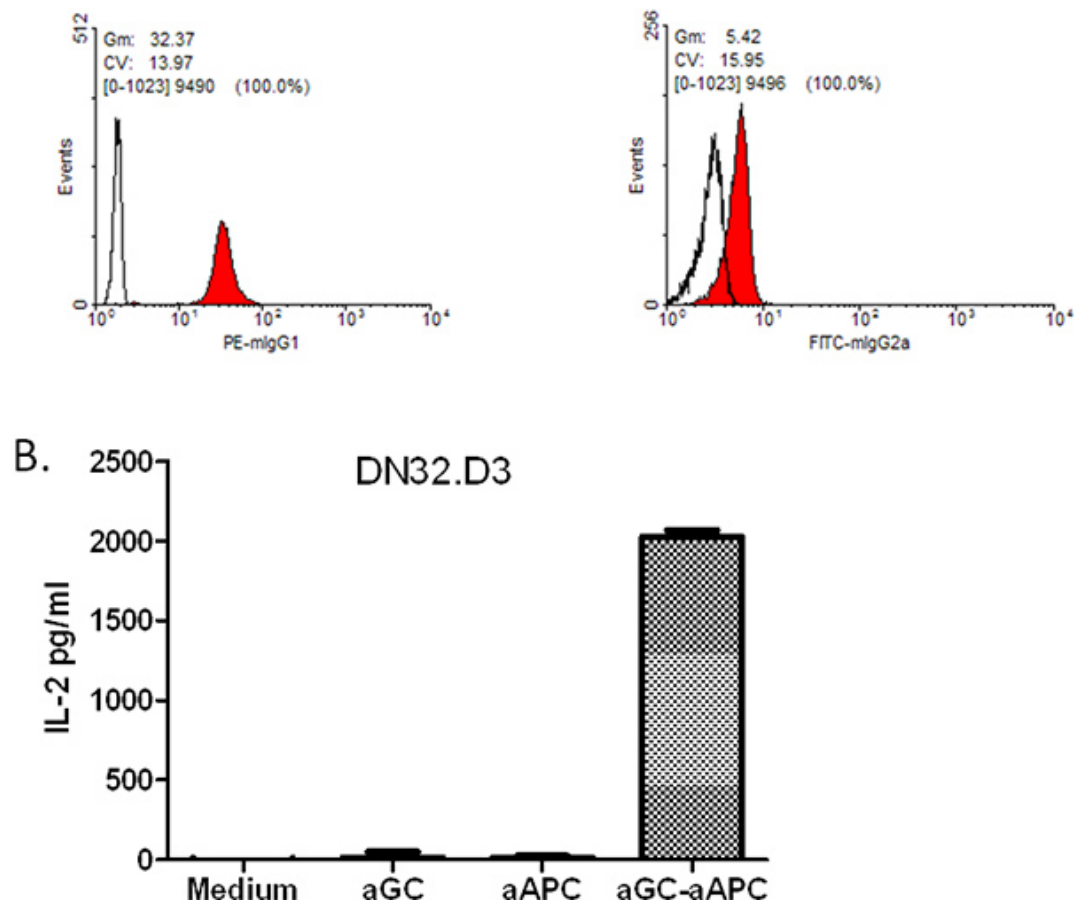

Figure 2. FACS staining of surface proteins on aAPCs. A) aAPCs were tested for the presence of CD1d:IgG dimer (via staining with PEconjugated anti-mouse IgG1) as well as anti-CD28 antibody (using FITC-conjugated anti-mouse IgG2a). Open histograms indicate isotype control; filled histograms represent the indicated antibodies. CD1d-lg Expressing aAPC can Stimulate IL-2 Production by NKT cells. B) The $\mathrm{Va}_{14}{ }^{+}$mouse NKT cell hybridoma, DN32.D3, was cocultured with either medium, soluble antigen ( $\alpha$-GalCer), unloaded aAPC or $\alpha-G a l C e r-$ loaded aAPC. Culture supernatants were harvested and standard sandwich ELISA was used to measure IL-2 production. 
A.

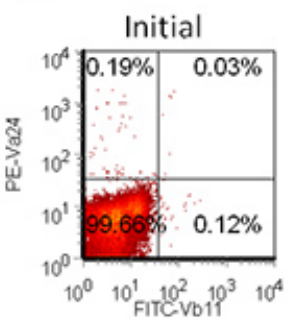

$\mathrm{CD}_{161}{ }^{+} \mathrm{CD}^{+}$

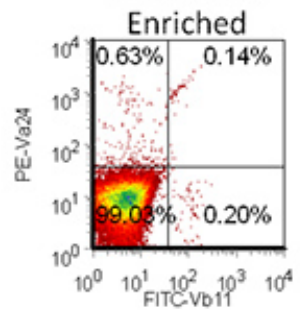

aAPC

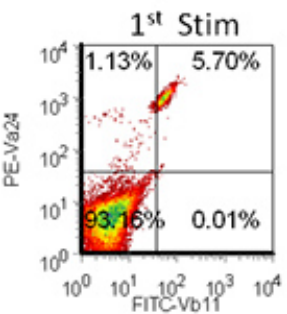

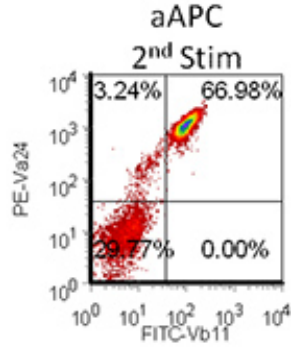

B.

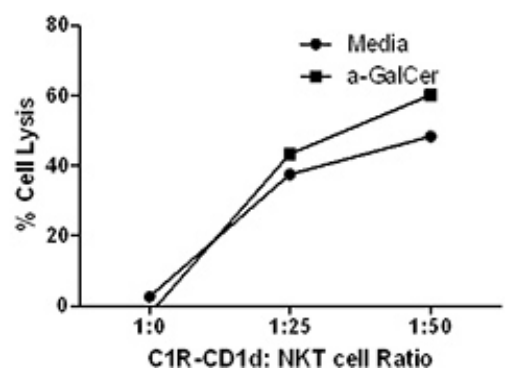

Figure 3. Expansion of NKT cells by CD1d-lg coated artificial antigen presenting cells. (A) Primary $C D 3^{+} \mathrm{CD} 161^{+}$double positive cells were isolated from PBMCs using magnetic separation. The sorted cells were stimulated with $\alpha$-GalCer loaded,CD1d-Ig coated aAPC for 14 days. The cells were stained for Va24 and V 311 following aAPC stimulation. (B) Primary human NKT cell mediated lysis of a B cell lymphoma line. C1RCD1d cells incubated with NKT cells at the indicated ratios in the presence or absence of antigen, a-GalCer (100 ng/ml) in 96-well U- bottom plates for 20-24 hr. NKT cell mediated cell lysis was assessed by by standard 51Cr-release assay.

A.

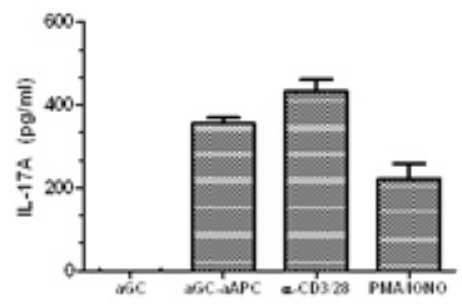

B.

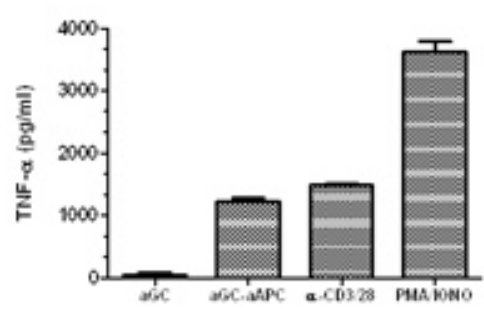

C.

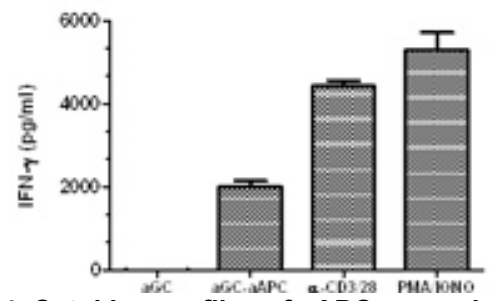

D.

Donor 1

initial
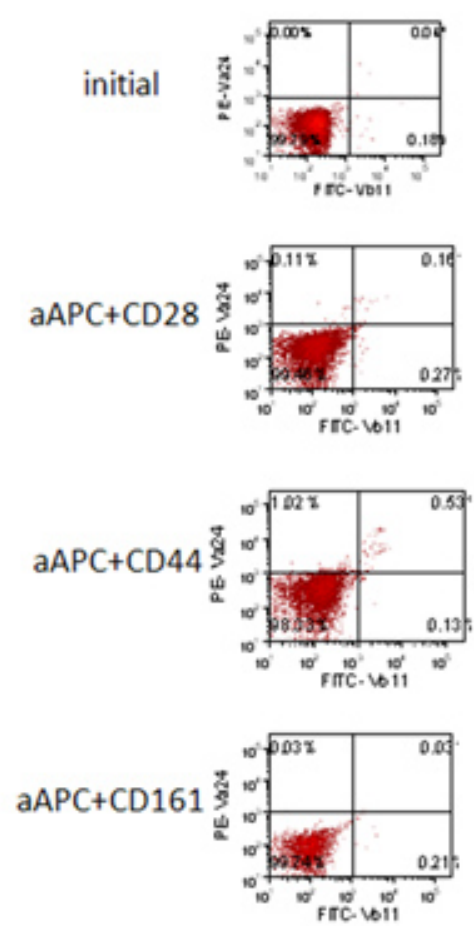

Donor 2
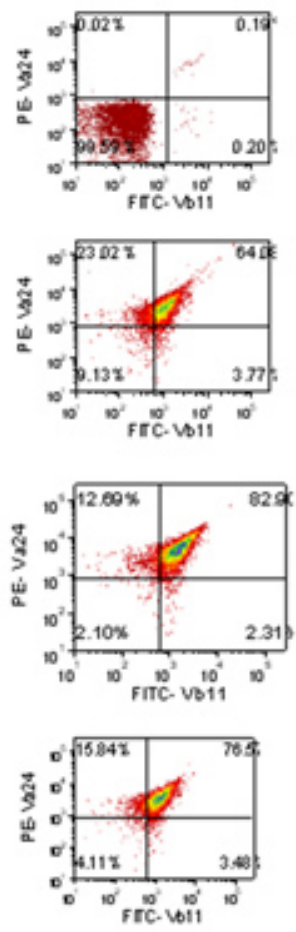

Figure 4. Cytokine profiles of aAPC-expanded NKT cells. After stimulation with a GalCer loaded aAPC for two weeks, the expanded NKT cells $\left(1 \times 10^{5} /\right.$ well $)$ were cocultured with soluble $\alpha$-GalCer, PMA/lonomycin, anti-CD3/28 microbeads, or $\alpha$-GalCer loaded aAPC $\left(2 \times 10^{5} /\right.$ well) for $48 \mathrm{hr}$. (A) IL-17A, (B) TNF- $\alpha$, and (C) IFN-y production was measured by standard cytokine ELISA. Data shown are net cytokine production after subtracting the negative controls (media and empty beads). (D) Primary T cells were isolated from PBMC using magnetic bead separation. The sorted cells were stimulated for two weeks with the indication a-GalCer loaded-aAPC. The cells were stained using Abs specific for Va24 ${ }^{+}$and $\mathrm{V} \beta 11^{+}$and analyzed by flow cytometry. 


\section{Discussion}

aAPC can be used to study the basic requirements for NKT cell activation and it has potential clinical value for ex vivo expansion of NK T cells for adoptive immunotherapy. Mescher et al. described one of the first bead based systems, where biotinylated murine MHC class I-peptide-single chain constructs were combined with biotinylated costimulatory molecules B7.1 and B7.2 via streptavidin to the surface of latex microspheres 14, 15. This approach has successfully been used to stimulate antigen-specific T cells from transgenic mice. In addition, since this approach uses a single chain MHC-peptide complex to ensure homogenous loading of the MHC molecules, each target peptide antigen would require a new transfection for expression of the desired single chain MHC-peptide complex, thus limiting the generality of the approach. Importantly, Dr. Schneck's group pioneered the bead based-aAPC, by developing another non-cellular bead based aAPC, made by coupling HLA-lg, signal 1 , and anti-CD28, signal 2, onto magnetic beads. HLA-Ig, a unique multimeric form of HLA fused to an immunoglobulin molecular scaffold ${ }^{16,17}$ was developed by his group. Subsequently, they developed MHC-lg based aAPC, which have been shown to effectively expand CMV and MART-1 specific CTL ${ }^{18}$. Here, we have demonstrated that CD1d-Ig based aAPC can be used to expand functional NKT cells. One study has used a similar system to examine the physical interaction of NK cells with CD1d ${ }^{19}$.

Notably, we have designed an artificial antigen presenting cell which is adaptable to any requirements we find necessary for optimal NKT cell proliferation. The aAPC expansion method provides a simple and reliable method for expanding and enriching human NKT cells. Our aAPC can be modified to systematically evaluate the role of a panel of potential costimulatory molecules and assess their role on NKT cell proliferation and function. Thus, aAPC represent a robust versatile technology useful for inducing and expanding NKT cells. The generation of aAPCs takes less than one week and is suitable for the production of large quantities of beads. However, a critical step in generating the aAPC is to confirm that CD1d-Ig is stably immobilized on the surface of the beads and to assess their functionality to ensure consistency from batch to batch. A potential limitation of the system is that there is not a mechanism in place to turn off stimulation, other than mechanical removal of the beads. Specifically, the engagement of the T cell receptor (TCR) with the antigen: CD1d/MHC complex typically generate the immunological synapse in concert with accessory/adhesion molecules, which can result in the induction of inhibitory or suppressive factors on both the T cell and antigen presenting cell. In the aAPC system, these factors may be upregulated by the T cell, but the bead will not express the cognate ligands for these receptors.

In addition, $\mathrm{CD}^{+} \mathrm{NKT}$ cells have been shown to suppress antitumor responses in mice and humans, therefore it is possible that nonselective activation of all NKT cells (i.e. global stimulation with $\alpha$-GalCer) or activation of the wrong subset could result in unwanted immunological outcomes. Consequently, one must phenotypically and functionally characterize the aAPC-expanded NKT cell population. As shown in Figure 4, we have found that stimulation with a-GalCer-loaded aAPC expressing anti-CD28 can result NKT cells producing Th1, Th2, and Th17 type cytokines. Murine studies have reported that challenge with IL-33, a recently identified cytokine, resulted in increased levels of circulating inflammatory cytokines such as IL-5 and IL-13. Treatment of NKT cells with IL-33 enhanced their cytokine production ${ }^{20}$. IL-33 is a specific ligand for ST2 and it has been shown that soluble ST2 can block IL-33 signaling. Thus, as an example of a future application, aAPC expressing ST2 could be generated and used to determine if one could selectively inhibit the production of Th2 cytokines while inducing Th1 cytokine secretion by NKT cells. It has also been reported that i.v. injection of Kb-expressing aAPC into C57BL/6 mice resulted in decreased lung metastasis of tumor ${ }^{21}$. Importantly, these data demonstrate that aAPC traffic to the lung and are able to activate effector T cell subsets. Therefore, one could generate multiple types of aAPC and examine the interplay between antigen specific $T$ cell subsets. To summarize, these studies demonstrate that CD1d-Ig based aAPC can be used to replace normal cellular APC, and have to the potential to enhance current clinical approaches for NKT cell based- adoptive immunotherapy.

\section{Disclosures}

No conflicts of interest declared.

\section{Acknowledgements}

The authors would like to thank Priyanka Subrahmanyam for helpful discussions. The authors have no competing financial interest. This work was supported by grants from the American Cancer Society, NIH/NCI K01 CA131487, R21 CA162273, R21 CA162277, and P30 Tumor Immunology and Immunotherapy Program to T.J. Webb. The content is solely the responsibility of the authors and does not necessarily represent the official views of the National Cancer Institute or the National Institutes of Health.

\section{References}

1. Fowlkes, B.J., et al. A novel population of T-cell receptor $\alpha \beta$-bearing thymocytes which predominantly expresses a single $V \beta$ gene family. Nature. 329, 251-254 (1987).

2. Prigozy, T.I., et al. Glycolipid antigen presentation by CD1d molecules. Science. 291, 664-667 (2001).

3. Davodeau, F., et al. Close phenotypic and functional similarities between human and murine $\alpha \beta$ T cells expressing invariant TCR $\alpha-c h a i n s . ~ J$. Immunol. 158, 5603-5611 (1997).

4. Exley, M., Garcia, J., Balk, S.P., \& Porcelli, S. Requirements for CD1d recognition by human invariant Va24 ${ }^{+} \mathrm{CD}^{-} \mathrm{CD}^{-} \mathrm{T}^{-}$cells. J. Exp. Med. 186, 109-120 (1997).

5. Koseki, H., et al. Dominant expression of a distinctive $\mathrm{V} 14^{+} \mathrm{T}$-cell antigen receptor $\alpha$ chain in mice. Proc. Natl. Acad. Sci. U.S.A. 88, 7518-7522 (1991).

6. Dellabona, P., Padovan, E., Casorati, G., Brockhaus, M., \& Lanzavecchia, A. An invariant Va24-JaQ/VB11 T cell receptor is expressed in all individuals by clonally expanded CD4-- $8^{-}$T cells. J. Exp. Med. 180, 1171-1176 (1994).

7. Porcelli, S.A. \& Modlin, R.L. The CD1 System: Antigen-presenting molecules for T cell recognition of lipids and glycolipids. Annu. Rev. Immunol. 17, 297-329 (1999). 
8. Harada, Y., et al. Expansion of alpha-galactosylceramide-stimulated Valpha24+ NKT cells cultured in the absence of animal materials. J. Immunother. 28, 314-321 (2005).

9. Bella, S.D., et al. Altered maturation of peripheral blood dendritic cells in patients with breast cancer. Br. J. Cancer. 89, 1463-1472 (2003).

10. Onishi, H., et al. Dysfunctional and Short-Lived Subsets in Monocyte-Derived Dendritic Cells from Patients with Advanced Cancer. Clinical Immunology. 105, 286-295 (2002).

11. Shimizu, K., et al. Evaluation of the function of human invariant NKT cells from cancer patients using alpha-galactosylceramide-loaded murine dendritic cells. J. Immunol. 177, 3484-3492 (2006).

12. Shiratsuchi, T., Schneck, J., Kawamura, A., \& Tsuji, M. Human CD1 dimeric proteins as indispensable tools for research on CD1-binding lipids and CD1-restricted T cells. Journal of immunological methods. 345, 49-59 (2009).

13. Webb, T.J., Bieler, J.G., Schneck, J.P., \& Oelke, M. Ex vivo induction and expansion of natural killer T cells by CD1d1-Ig coated artificial antigen presenting cells. J. Immunol. Methods. 346, 38-44 (2009).

14. Tham, E.L., Jensen, P.L., \& Mescher, M.F. Activation of antigen-specific T cells by artificial cell constructs having immobilized multimeric peptide-class I complexes and recombinant B7-Fc proteins. J. Immunol. Methods. 249, 111-119 (2001).

15. Goldberg, J., Shrikant, P., \& Mescher, M.F. In vivo augmentation of tumor-specific CTL responses by class I/peptide antigen complexes on microspheres (large multivalent immunogen). J. Immunol. 170, 228-235 (2003).

16. Dal Porto, J., et al. A soluble divalent class I major histocompatibility complex molecule inhibits alloreactive T cells at nanomolar concentrations. Proc. Natl. Acad. Sci. U.S.A. 90, 6671-6675 (1993).

17. Greten, T.F., et al. Direct visualization of antigen-specific T cells: HTLV-1 Tax11-19-specific CD8+ T cells are activated in peripheral blood and accumulate in cerebrospinal fluid from HAM/TSP patients. PNAS. 95, 7568-7573 (1998).

18. Oelke, M., et al. Ex vivo induction and expansion of antigen-specific cytotoxic T cells by HLA-lg-coated artificial antigen-presenting cells. Nat. Med. 9, 619-625 (2003).

19. Huang, M.M.S., Borszcz, P., Sidobre, S., Kronenberg, M., \& Kane, K.P. CD1d1 Displayed on Cell Size Beads Identifies and Enriches an NK Cell Population Negatively Regulated by CD1d1. J. Immunol. 172, 5304-5312 (2004).

20. Bourgeois, E., et al. The pro-Th2 cytokine IL-33 directly interacts with invariant NKT and NK cells to induce IFN-gamma production. Eur. J. Immunol. 39, 1046-1055 (2009).

21. Ugel, S., et al. In vivo administration of artificial antigen-presenting cells activates low-avidity T cells for treatment of cancer. Cancer Res. $\mathbf{6 9}$, 9376-9384 (2009). 